\title{
The Yang-Laplace Transform for Solving the IVPs with Local Fractional Derivative
}

\author{
Chun-Guang Zhao, ${ }^{1}$ Ai-Min Yang, ${ }^{2,3}$ Hossein Jafari, ${ }^{4}$ and Ahmad Haghbin ${ }^{4}$ \\ ${ }^{1}$ Department of Mathematics, Handan College, Handan, Hebei 056004, China \\ ${ }^{2}$ College of Science, Hebei United University, Tangshan 063009, China \\ ${ }^{3}$ College of Mechanical Engineering, Yanshan University, Qinhuangdao 066004, China \\ ${ }^{4}$ Department of Mathematics, Faculty of Mathematical Sciences, University of Mazandaran, Babolsar 47415-416, Iran
}

Correspondence should be addressed to Ai-Min Yang; aimin_heut@163.com

Received 25 October 2013; Accepted 7 November 2013; Published 8 January 2014

Academic Editor: Abdon Atangana

Copyright ( 2014 Chun-Guang Zhao et al. This is an open access article distributed under the Creative Commons Attribution License, which permits unrestricted use, distribution, and reproduction in any medium, provided the original work is properly cited.

The IVPs with local fractional derivative are considered in this paper. Analytical solutions for the homogeneous and nonhomogeneous local fractional differential equations are discussed by using the Yang-Laplace transform.

\section{Introduction}

In recent years, the ordinary and partial differential equations have found applications in many problems in mathematical physics [1, 2]. Initial value problems (IVPs) for ordinary and partial differential equations have been developed by some authors in [3-6]. There are analytical methods and numerical methods for solving the differential equations, such as the finite element method [6], the harmonic wavelet method [7-9], the Adomian decomposition method [10-12], the homotopy analysis method $[13,14]$, the homotopy decomposition method $[15,16]$, the heat balance integral method $[17,18]$, the homotopy perturbation method [19], the variational iteration method [20], and other methods [21].

Recently, owing to limit of classical and fractional differential equations, the local fractional differential equations have been applied to describe nondifferentiable problems for the heat and wave in fractal media [22, 23], the structure relation in fractal elasticity [24], and Fokker-Planck equation in fractal media [25]. Some methods were utilized to solve the local fractional differential equations. For example, the local fractional variation iteration method was used to solve the heat conduction in fractal media [26, 27]. The local fractional decomposition method for solving the local fractional diffusion and heat-conduction equations was considered in $[28,29]$. The local fractional series expansion method for solving the Schrödinger equation with the local fractional derivative was presented [30]. The Yang-Laplace transform structured in 2011 [22] was suggested to deal with local fractional differential equations [31, 32]. The coupling method for variational iteration method within Yang-Laplace transform for solving the heat conduction in fractal media was proposed in [33].

In this paper, our aim is to use the Yang-Laplace transform to solve IVPs with local fractional derivative. The structure of the paper is as follows. In Section 2, some definitions and properties for the Yang-Laplace transform are given. Section 3 is devoted to the solutions for the homogeneous and nonhomogeneous IVPs with local fractional derivative. Finally, conclusions are presented in Section 4.

\section{Yang-Laplace Transform}

In this section we show some definitions and properties for the Yang-Laplace transform.

The local fractional integral operator is defined as $[22,23,26-33]$

$$
\begin{aligned}
{ }_{a} I_{b}{ }^{(\alpha)} f(x) & =\frac{1}{\Gamma(1+\alpha)} \int_{a}^{b} f(t)(d t)^{\alpha} \\
& =\frac{1}{\Gamma(1+\alpha)} \lim _{\Delta t \rightarrow 0} \sum_{j=0}^{j=N-1} f\left(t_{j}\right)\left(\Delta t_{j}\right)^{\alpha},
\end{aligned}
$$


where $\Delta t_{j}=t_{j+1}-t_{j}, \Delta t=\max \left\{\Delta t_{0}, \Delta t_{1}, \ldots, \Delta t_{j}, \ldots\right\}$, $\left[t_{j}, t_{j+1}\right], j=0, \ldots, N-1, t_{0}=a, t_{N}=b$, is a partition of the interval $[a, b]$.

As the inverse operator of (1), the local fractional derivative operator is given by $[22,23,26-33]$

$$
f^{(\alpha)}\left(x_{0}\right)=\left.\frac{d^{\alpha} f(x)}{d x^{\alpha}}\right|_{x=x_{0}}=\lim _{x \rightarrow x_{0}} \frac{\Delta^{\alpha}\left(f(x)-f\left(x_{0}\right)\right)}{\left(x-x_{0}\right)^{\alpha}},
$$

with $\Delta^{\alpha}\left(f(x)-f\left(x_{0}\right)\right) \cong \Gamma(1+\alpha) \Delta\left(f(x)-f\left(x_{0}\right)\right)$.

The Yang-Laplace transform is expressed by [22, 31-33]

$\tilde{L}_{\alpha}\{f(x)\}=f_{s}^{\widetilde{L}, \alpha}(s)=\frac{1}{\Gamma(1+\alpha)} \int_{0}^{\infty} E_{\alpha}\left(-s^{\alpha} x^{\alpha}\right) f(x)(d x)^{\alpha}$,

$$
0<\alpha \leq 1
$$

where $f(x)$ is a local fractional continuous function.

The inverse Yang-Laplace transform reads as [22, 31-33]

$$
\begin{aligned}
f(x)= & \widetilde{L}_{\alpha}^{-1}\left\{f_{s}^{L, \alpha}(s)\right\}=\frac{1}{(2 \pi)^{\alpha}} \\
& \times \int_{\beta-i \infty}^{\beta+i \infty} E_{\alpha}\left(s^{\alpha} x^{\alpha}\right) f_{s}^{\widetilde{L}, \alpha}(s)(d s)^{\alpha},
\end{aligned}
$$

where $s^{\alpha}=\beta^{\alpha}+i^{\alpha} \infty^{\alpha}$ and $\operatorname{Re}\left(s^{\alpha}\right)=\beta^{\alpha}$.

Some properties for Yang-Laplace transform are presented as follows [21, 22, 22-33]:

$$
\begin{gathered}
\widetilde{L}_{\alpha}\{a f(x)+b g(x)\}=a \widetilde{L}_{\alpha}\{f(x)\}+b \widetilde{L}_{\alpha}\{g(x)\}, \\
\widetilde{L}_{\alpha}\left\{f^{(n \alpha)}(x)\right\}=s^{n \alpha} \widetilde{L}_{\alpha}\{f(x)\}-\sum_{k=1}^{n} s^{(k-1) \alpha} f^{(n-k) \alpha}(0), \\
\lim _{x \rightarrow 0} f(x)=\lim _{s \rightarrow \infty} s^{\alpha} F(s), \\
\lim _{x \rightarrow \infty} f(x)=\lim _{s \rightarrow 0} s^{\alpha} F(s), \\
\widetilde{L}_{\alpha}\{f(a x)\}=\frac{1}{a^{\alpha}} f_{s}^{L, \alpha}\left(\frac{s}{a}\right), \quad a>0, \\
\widetilde{L}_{\alpha}\left\{x^{k \alpha} f(x)\right\}=(-1)^{k} \frac{d^{k \alpha} f_{s}^{L, \alpha}(s)}{d s^{k \alpha}}, \\
\widetilde{L}_{\alpha}\{f(x-c)\}=f_{s}^{L, \alpha}(s) E_{\alpha}\left(-c^{\alpha} s^{\alpha}\right), \\
\widetilde{L}_{\alpha}\left\{f(x) E_{\alpha}\left(c^{\alpha} x^{\alpha}\right)\right\}=f_{s}^{L, \alpha}(s-c), \\
\widetilde{L}_{\alpha}\left\{x^{k \alpha} E_{\alpha}\left(c^{\alpha} x^{\alpha}\right)\right\}=\frac{\Gamma(1+k \alpha)}{(s-c)^{(k+1) \alpha}} \\
\widetilde{L}_{\alpha}\left\{\sin _{\alpha}\left(c^{\alpha} x^{\alpha}\right)\right\}=\frac{c^{\alpha}}{s^{2 \alpha}+c^{2 \alpha}} \\
\widetilde{L}_{\alpha}\left\{\cos _{\alpha}\left(c^{\alpha} x^{\alpha}\right)\right\}=\frac{s^{\alpha}}{s^{2 \alpha}+c^{2 \alpha}} \\
\widetilde{L}_{\alpha}\left\{x^{k \alpha}\right\}=\frac{\Gamma(1+k \alpha)}{s^{(k+1) \alpha}} .
\end{gathered}
$$

\section{IVPs with Local Fractional Derivatives}

In this section we handle the homogeneous and nonhomogeneous IVPs with local fractional derivative.

\subsection{Homogeneous IVPs with Local Fractional Derivative}

Example 1. The homogeneous IVPs with local fractional derivative are expressed by

$$
\frac{d^{2 \alpha} y}{d^{2 \alpha} x}-\frac{d^{\alpha} y}{d^{\alpha} x}+2 y=0
$$

The initial boundary conditions are presented as

$$
y(0)=1, \quad y^{(\alpha)}(0)=0 .
$$

From (6) we have

$$
\begin{gathered}
\widetilde{L}_{\alpha}\left\{y^{(\alpha)}(x)\right\}=s^{\alpha} \widetilde{L}_{\alpha}\{y(x)\}-y(0), \\
\widetilde{L}_{\alpha}\left\{y^{(2 \alpha)}(x)\right\}=s^{2 \alpha} \widetilde{L}_{\alpha}\{y(x)\}-s^{\alpha} y(0)-f^{(\alpha)}(0) .
\end{gathered}
$$

Hence, making use of (19) and (20), (19) can be written as

$$
\begin{aligned}
& s^{2 \alpha} \widetilde{L}_{\alpha}\{y(x)\}-s^{\alpha} y(0)-f^{(\alpha)}(0)-\left\{s^{\alpha} \widetilde{L}_{\alpha}\{y(x)\}-y(0)\right\} \\
& +2 \widetilde{L}_{\alpha}\{y(x)\}=0 .
\end{aligned}
$$

Hence, we obtain

$$
\widetilde{L}_{\alpha}\{y(x)\}=\frac{1}{s^{\alpha}+2} y(0)=\frac{1}{s^{\alpha}+2} .
$$

So, making use of (13), we get the solution of (17):

$$
y(x)=E_{\alpha}\left(-2 x^{\alpha}\right) .
$$

The solution of (17) for $\alpha=\ln 2 / \ln 3$ is shown in Figure 1 .

Example 2. Let us consider the homogeneous IVPs with local fractional derivative in the form

$$
\frac{d^{4 \alpha} y}{d^{4 \alpha} x}-y=0
$$

subject to initial boundary conditions

$$
\begin{gathered}
y(0)=0, \quad y^{(\alpha)}(0)=0, \\
y^{(2 \alpha)}(0)=0, \quad y^{(3 \alpha)}(0)=1 .
\end{gathered}
$$

From (6) we have

$$
\begin{aligned}
\widetilde{L}_{\alpha}\left\{y^{(4 \alpha)}(x)\right\}= & s^{4 \alpha} \widetilde{L}_{\alpha}\{y(x)\}-s^{3 \alpha} y(0)-s^{2 \alpha} y^{(\alpha)}(0) \\
& -s^{\alpha} y^{(2 \alpha)}(0)-f^{(3 \alpha)}(0),
\end{aligned}
$$

so that

$$
\begin{gathered}
s^{4 \alpha} \widetilde{L}_{\alpha}\{y(x)\}-s^{3 \alpha} y(0)-s^{2 \alpha} y^{(\alpha)}(0)-s^{\alpha} y^{(2 \alpha)}(0) \\
-f^{(3 \alpha)}(0)-\widetilde{L}_{\alpha}\{y(x)\}=0 .
\end{gathered}
$$




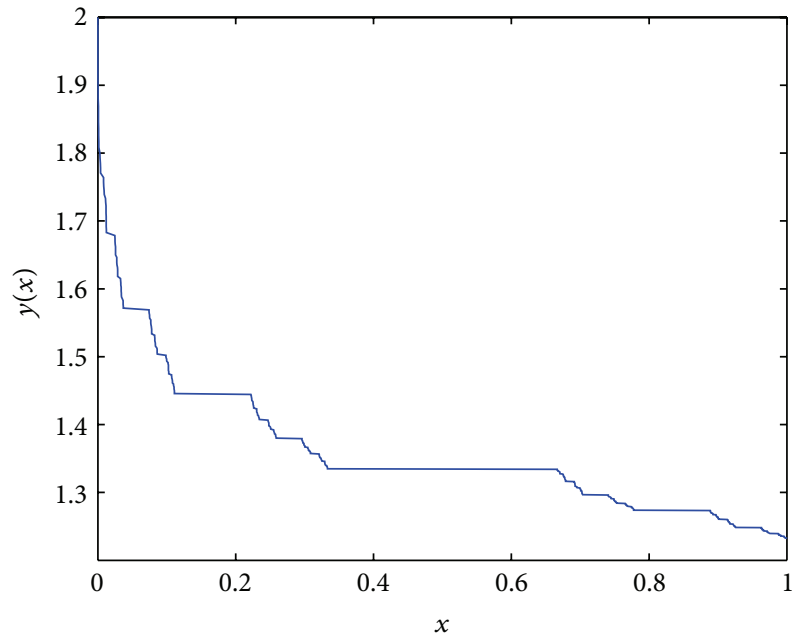

FIGURE 1: Graph of $y(x)$ for $\alpha=\ln 2 / \ln 3$.

Hence, (27) can be written as

$$
s^{4 \alpha} \widetilde{L}_{\alpha}\{y(x)\}-1-\widetilde{L}_{\alpha}\{y(x)\}=0,
$$

which leads to

$$
\widetilde{L}_{\alpha}\{y(x)\}=\frac{1}{s^{4 \alpha}-1}
$$

Therefore, we get

$$
\begin{aligned}
y(x) & =\widetilde{L}_{\alpha}^{-1}\left\{\frac{1}{s^{4 \alpha}-1}\right\} \\
& =\widetilde{L}_{\alpha}^{-1}\left\{\frac{1}{2}\left(\frac{1}{2} \frac{1}{s^{\alpha}-1}-\frac{1}{2} \frac{1}{s^{\alpha}+1}-\frac{1}{s^{2 \alpha}+1}\right)\right\} \\
& =\frac{1}{4} E_{\alpha}\left(-x^{\alpha}\right)-\frac{1}{4} E_{\alpha}\left(x^{\alpha}\right)-\frac{1}{2} \sin _{\alpha}\left(x^{\alpha}\right)
\end{aligned}
$$

The exact solution of (24) for $\alpha=\ln 2 / \ln 3$ is shown in Figure 2.

\subsection{Nonhomogeneous IVPs with Local Fractional Derivative}

Example 3. We now consider the non-homogeneous IVPs with local fractional derivative

$$
\frac{d^{2 \alpha} y}{d^{2 \alpha} x}-y=\sin _{\alpha}\left(x^{\alpha}\right)
$$

subject to initial boundary conditions

$$
y(0)=0, \quad y^{(\alpha)}(0)=1
$$

By using (6), we have

$$
\begin{gathered}
\widetilde{L}_{\alpha}\left\{y^{(2 \alpha)}(x)\right\}=s^{2 \alpha} \widetilde{L}_{\alpha}\{y(x)\}-s^{\alpha} y(0)-f^{(\alpha)}(0), \\
\widetilde{L}_{\alpha}\left\{\sin _{\alpha}\left(x^{\alpha}\right)\right\}=\frac{1}{s^{2 \alpha}+1}
\end{gathered}
$$

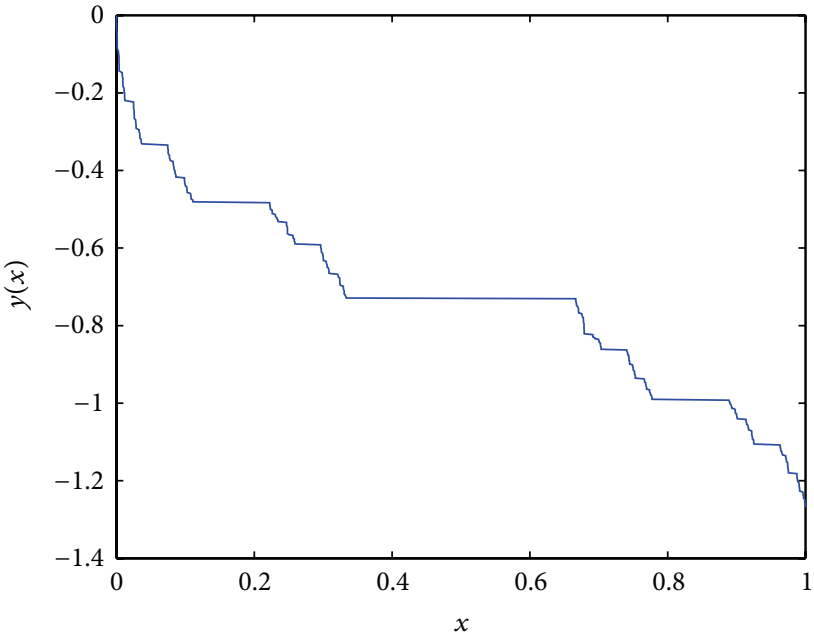

FIGURE 2: Graph of $y(x)$ for $\alpha=\ln 2 / \ln 3$.

so that

$$
\widetilde{L}_{\alpha}\{y(x)\}=\frac{3}{4}\left(\frac{1}{s^{\alpha}-1}-\frac{1}{s^{\alpha}+1}\right)-\frac{1}{2} \frac{1}{s^{2 \alpha}+1} .
$$

So,

$$
y(x)=\frac{3}{4} E_{\alpha}\left(-x^{\alpha}\right)-\frac{3}{4} E_{\alpha}\left(x^{\alpha}\right)-\frac{1}{2} \sin _{\alpha}\left(x^{\alpha}\right) .
$$

The exact solution of (31) for $\alpha=\ln 2 / \ln 3$ is shown in Figure 3.

Example 4. The non-homogeneous IVPs with local fractional derivative are

$$
\frac{d^{2 \alpha} y}{d^{2 \alpha} x}+y=E_{\alpha}\left(x^{\alpha}\right) .
$$

The initial boundary conditions are

$$
y(0)=1, \quad y^{(\alpha)}(0)=0 .
$$

In view of (6), we give

$$
\widetilde{L}_{\alpha}\{y(x)\}=\frac{1}{\left(s^{\alpha}+1\right)\left(s^{2 \alpha}+1\right)}+\frac{s^{\alpha}}{s^{2 \alpha}+1} .
$$

So, we obtain

$$
\begin{aligned}
y(x)= & \cos _{\alpha}\left(x^{\alpha}\right)+\frac{1}{\Gamma(1+\alpha)} \int_{0}^{x} E_{\alpha}(x-t)^{\alpha} \sin _{\alpha}\left(t^{\alpha}\right)(d t)^{\alpha} \\
= & \cos _{\alpha}\left(x^{\alpha}\right)+\frac{1}{\Gamma(1+\alpha)} \\
& \times \int_{0}^{x} E_{\alpha}\left(t^{\alpha}\right)\left(\sin _{\alpha}\left(x^{\alpha}\right) \cos _{\alpha}\left(t^{\alpha}\right)\right. \\
& \left.\quad-\cos _{\alpha}\left(x^{\alpha}\right) \sin _{\alpha}\left(t^{\alpha}\right)\right)(d t)^{\alpha}
\end{aligned}
$$




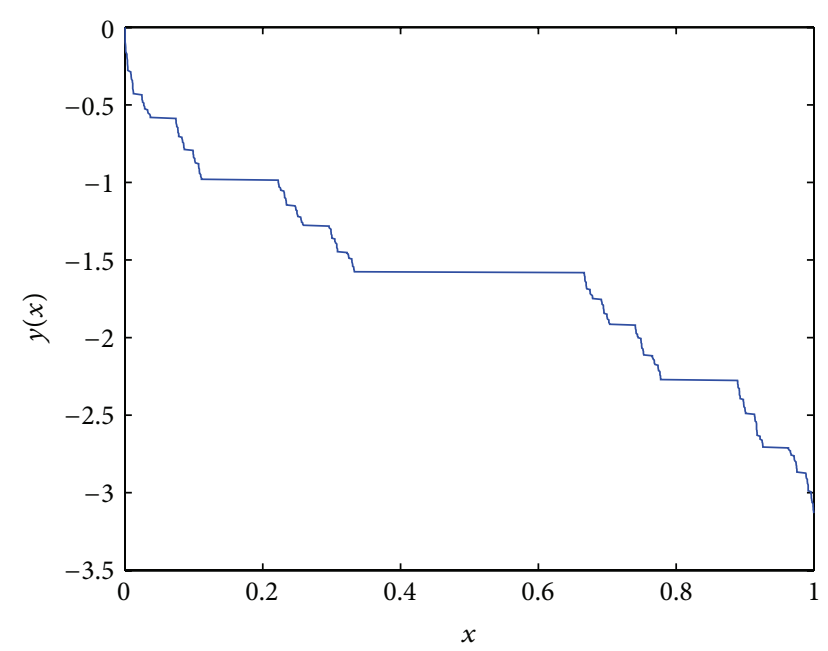

FIGURE 3: Graph of $y(x)$ for $\alpha=\ln 2 / \ln 3$.

$$
\begin{aligned}
= & \cos _{\alpha}\left(x^{\alpha}\right) \\
& +\sin _{\alpha}\left(x^{\alpha}\right)\left\{\frac{1}{\Gamma(1+\alpha)} \int_{0}^{x} E_{\alpha}\left(t^{\alpha}\right) \cos _{\alpha}\left(t^{\alpha}\right)(d t)^{\alpha}\right\} \\
& -\cos _{\alpha}\left(x^{\alpha}\right)\left\{\frac{1}{\Gamma(1+\alpha)} \int_{0}^{x} E_{\alpha}\left(t^{\alpha}\right) \sin _{\alpha}\left(t^{\alpha}\right)(d t)^{\alpha}\right\} \\
= & \cos _{\alpha}\left(x^{\alpha}\right) \\
& +\frac{\sin _{\alpha}\left(x^{\alpha}\right)\left\{E_{\alpha}\left(x^{\alpha}\right)\left[\cos _{\alpha}\left(x^{\alpha}\right)+\sin \left(x^{\alpha}\right)\right]-1\right\}}{2} \\
& -\frac{\cos _{\alpha}\left(x^{\alpha}\right)\left\{E_{\alpha}\left(x^{\alpha}\right)\left[\sin _{\alpha}\left(x^{\alpha}\right)-\cos _{\alpha}\left(x^{\alpha}\right)\right]+1\right\}}{2} \\
= & \frac{1}{2}\left[\cos _{\alpha}\left(x^{\alpha}\right)-\sin _{\alpha}\left(x^{\alpha}\right)+E_{\alpha}\left(x^{\alpha}\right)\right] .
\end{aligned}
$$

The exact solution of (36) for $\alpha=\ln 2 / \ln 3$ is shown in Figure 4.

\section{Conclusions}

In this work we have used the Yang-Laplace transform to handle the homogeneous and non-homogeneous IVPs with looselocal fractional derivative. Some illustrative examples of approximate solutions for local fractional IVPs are discussed. The nondifferentiable solutions for fractal dimension $\alpha=$ $\ln 2 / \ln 3$ are shown graphically. The obtained results illustrate that the Yang-Laplace transform is an efficient mathematical tool to solve the homogeneous and non-homogeneous IVPs with local fractional derivative.

\section{Conflict of Interests}

The authors declare that there is no conflicts of interests regarding publication of this paper.

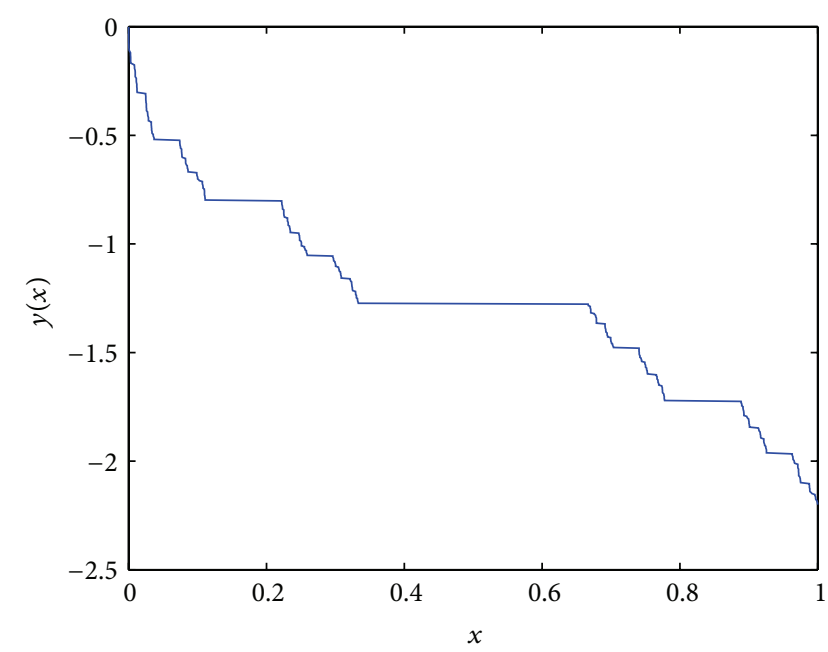

FIGURE 4: Graph of $y(x)$ for $\alpha=\ln 2 / \ln 3$.

\section{Acknowledgments}

This work was supported by national scientific and technological support projects (no. 2012BAE09B00), the national natural Science Foundation of China (no. 61202259 and no. 61170317), the national natural Science Foundation of Hebei Province (no. A2012209043 and no. E2013209215), and Hebei province of China Scientific Research Subject in Twelfth FiveYear Plan (no. 13090074).

\section{References}

[1] N. S. Koshlyakov, M. M. Smirnov, and E. B. Gliner, Differential Equations of Mathematical Physics, North-Holland, New York, NY, USA, 1964.

[2] U. Tyn Myint, Partial Differential Equations of Mathematical Physics, Elsevier, New York, NY, USA, 1973.

[3] A. H. Stroud, "Initial value problems for ordinary differential equations," in Numerical Quadrature and Solution of Ordinary Differential Equations, pp. 207-303, Springer, New York, NY, USA, 1974.

[4] H. Rutishauser, "Initial value problems for ordinary differential equations," in Lectures on Numerical Mathematics, pp. 208-277, Birkhäuser, Boston, Mass, USA, 1990.

[5] J. A. Gatica, V. Oliker, and P. Waltman, "Singular nonlinear boundary value problems for second-order ordinary differential equations," Journal of Differential Equations, vol. 79, no. 1, pp. $62-78,1989$.

[6] S. F. Davis and J. E. Flaherty, "An adaptive finite element method for initial-boundary value problems for partial differential equations," SIAM Journal on Scientific and Statistical Computing, vol. 3, no. 1, pp. 6-27, 1982.

[7] C. Cattani and A. Kudreyko, "Harmonic wavelet method towards solution of the Fredholm type integral equations of the second kind," Applied Mathematics and Computation, vol. 215, no. 12, pp. 4164-4171, 2010.

[8] C. Cattani, "Harmonic wavelet solutions of the Schrödinger equation," International Journal of Fluid Mechanics Research, vol. 30, no. 5, pp. 463-472, 2003. 
[9] C. Cattani, "Harmonic wavelets towards the solution of nonlinear PDE," Computers \& Mathematics with Applications, vol. 50, no. 8-9, pp. 1191-1210, 2005.

[10] V. Daftardar-Gejji and H. Jafari, "Adomian decomposition: a tool for solving a system of fractional differential equations," Journal of Mathematical Analysis and Applications, vol. 301, no. 2, pp. 508-518, 2005.

[11] J. S. Duan, R. Rach, and A. M. Wazwaz, "Solution of the model of beam-type micro- and nano-scale electrostatic actuators by a new modified Adomian decomposition method for nonlinear boundary value problems," International Journal of Non-Linear Mechanics, vol. 49, pp. 159-169, 2013.

[12] C. Li and Y. Wang, "Numerical algorithm based on Adomian decomposition for fractional differential equations," Computers \& Mathematics with Applications, vol. 57, no. 10, pp. 1672-1681, 2009.

[13] H. Jafari and S. Seifi, "Homotopy analysis method for solving linear and nonlinear fractional diffusion-wave equation," Communications in Nonlinear Science and Numerical Simulation, vol. 14, no. 5, pp. 2006-2012, 2009.

[14] I. Hashim, O. Abdulaziz, and S. Momani, "Homotopy analysis method for fractional IVPs," Communications in Nonlinear Science and Numerical Simulation, vol. 14, no. 3, pp. 674-684, 2009.

[15] A. Atangana and A. Secer, "The time-fractional coupledKorteweg-de-Vries equations," Abstract and Applied Analysis, vol. 2013, Article ID 947986, 8 pages, 2013.

[16] A. Atangana and A. K1lıçman, "Analytical solutions of boundary values problem of $2 \mathrm{D}$ and $3 \mathrm{D}$ poisson and biharmonic equations by homotopy decomposition method," Abstract and Applied Analysis, vol. 2013, Article ID 380484, 9 pages, 2013.

[17] J. Hristov, "Heat-balance integral to fractional (half-time) heat diffusion sub-model," Thermal Science, vol. 14, no. 2, pp. 291-316, 2010.

[18] J. Hristov, “Transient flow of a generalized second grade fluid due to a constant surface shear stress: an approximate integralbalance solution," International Review of Chemical Engineering, vol. 3, no. 6, pp. 802-809, 2011.

[19] S. T. Mohyud-Din and M. A. Noor, "Homotopy perturbation method for solving fourth-order boundary value problems," Mathematical Problems in Engineering, vol. 2007, Article ID 98602, 15 pages, 2007.

[20] M. A. Noor and S. T. Mohyud-Din, "Variational iteration method for solving higher-order nonlinear boundary value problems using He's polynomials," International Journal of Nonlinear Sciences and Numerical Simulation, vol. 9, no. 2, pp. 141-156, 2008.

[21] D. Baleanu, K. Diethelm, E. Scalas, and J. J. Trujillo, Fractional Calculus Models and Numerical Methods, vol. 3 of Series on Complexity, Nonlinearity and Chaos, World Scientific Publishing, Boston, Mass, USA, 2012.

[22] X.-J. Yang, Local Fractional Functional Analysis and Its Applications, Asian Academic Publisher, Hong Kong, 2011.

[23] X. J. Yang, Advanced Local Fractional Calculus and Its Applications, World Science Publisher, New York, NY, USA, 2012.

[24] A. Carpinteri, B. Chiaia, and P. Cornetti, "The elastic problem for fractal media: basic theory and finite element formulation," Computers and Structures, vol. 82, no. 6, pp. 499-508, 2004.

[25] K. M. Kolwankar and A. D. Gangal, "Local fractional FokkerPlanck equation," Physical Review Letters, vol. 80, no. 2, pp. 214217, 1998.
[26] X. J. Yang and D. Baleanu, "Fractal heat conduction problem solved by local fractional variation iteration method," Thermal Science, vol. 17, no. 2, pp. 625-628, 2013.

[27] J. H. He and F. J. Liu, "Local fractional variational iteration method for fractal heat transfer in silk cocoon hierarchy," Nonlinear Science Letters A, vol. 4, no. 1, pp. 15-20, 2013.

[28] X.-J. Yang, D. Baleanu, and W. P. Zhong, "Approximate solutions for diffusion equations on cantor space-time," Proceedings of the Romanian Academy A, vol. 14, no. 2, pp. 127-133, 2013.

[29] X. J. Yang, D. Baleanu, M. P. Lazarevic, and M. S. Cajic, "Fractal boundary value problems for integral and differential equations with local fractional operators," Thermal Science, pp. 103-103, 2013.

[30] Y. Zhao, D.-F. Cheng, and X.-J. Yang, "Approximation solutions for local fractional Schrödinger equation in the onedimensional Cantorian system," Advances in Mathematical Physics, vol. 2013, Article ID 291386, 5 pages, 2013.

[31] J.-H. He, "Asymptotic methods for solitary solutions and compactons," Abstract and Applied Analysis, vol. 2012, Article ID 916793, 130 pages, 2012.

[32] W. P. Zhong and F. Gao, "Application of the Yang-Laplace transforms to solution to nonlinear fractional wave equation with fractional derivative," in Proceeding of the $3 r d$ International Conference on Computer Technology and Development, pp. 209213, 2011.

[33] C. F. Liu, S. S. Kong, and S. J. Yuan, "Reconstructive schemes for variational iteration method within Yang-Laplace transform with application to fractal heat conduction problem," Thermal Science, vol. 17, no. 3, pp. 715-721, 2013. 


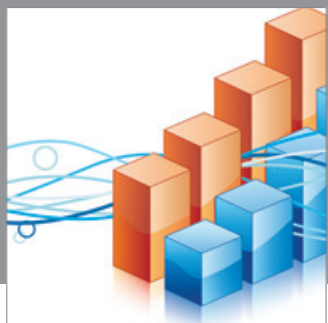

Advances in

Operations Research

mansans

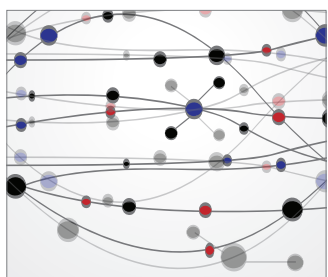

The Scientific World Journal
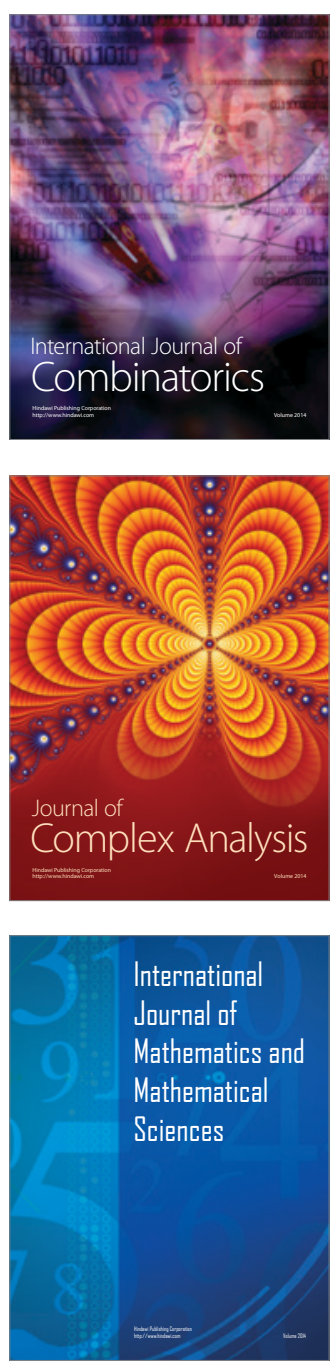
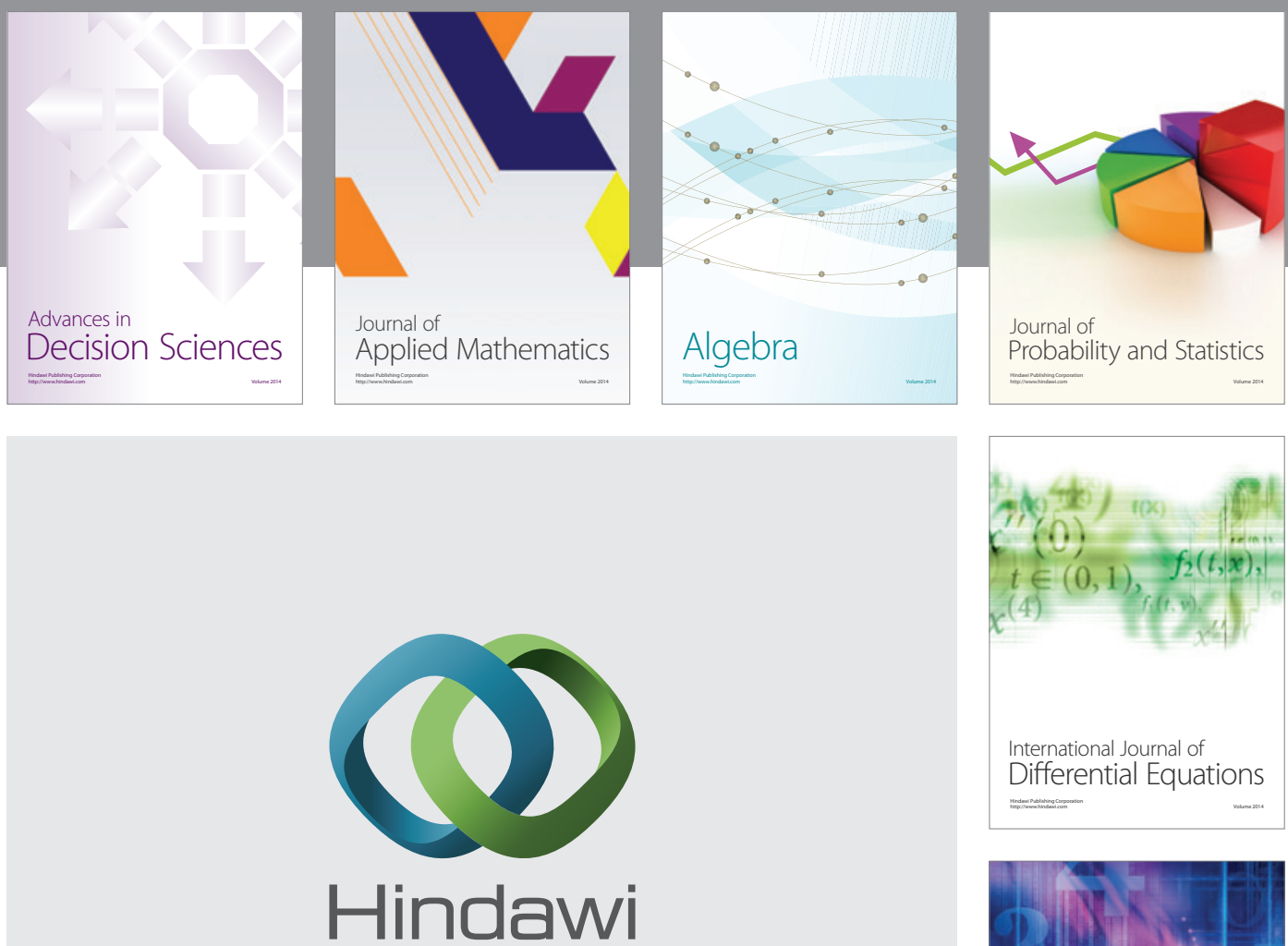

Submit your manuscripts at http://www.hindawi.com
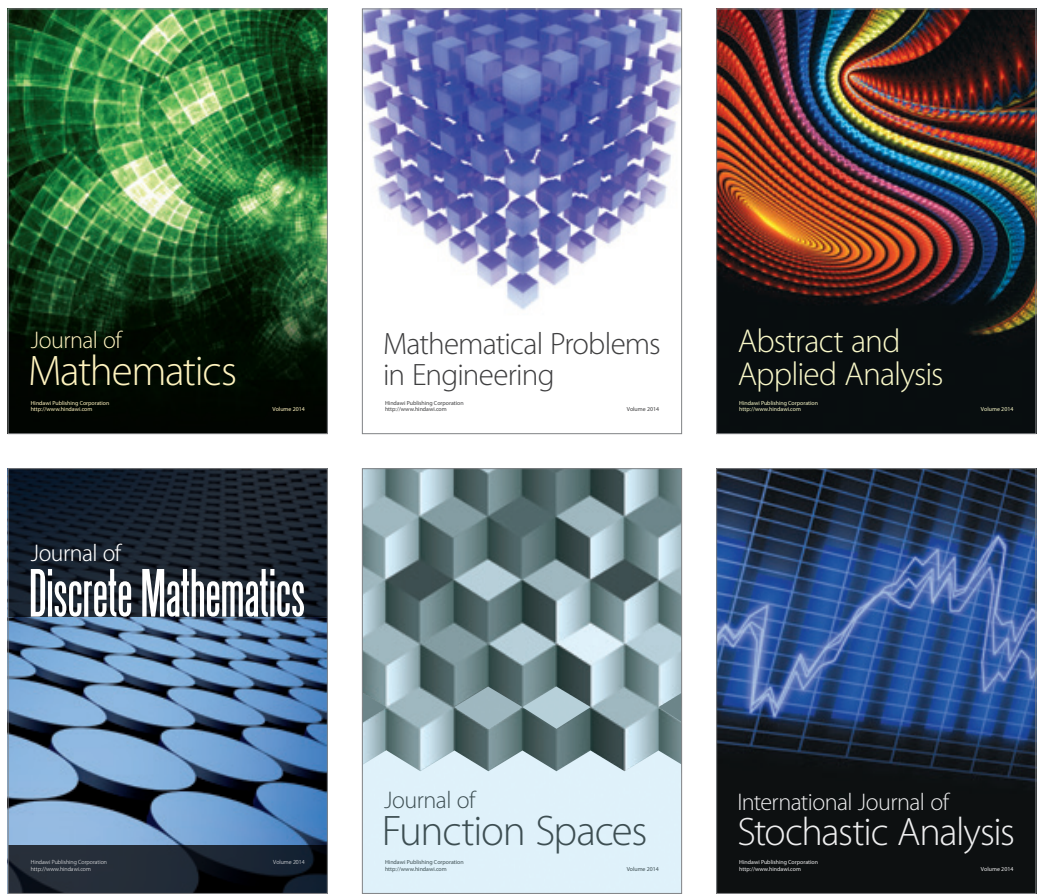

Journal of

Function Spaces

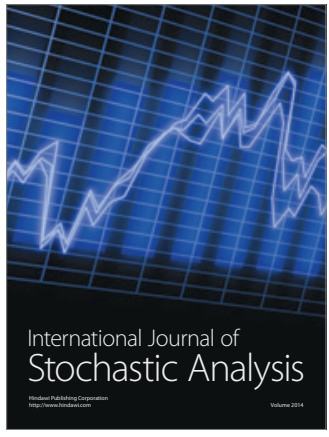

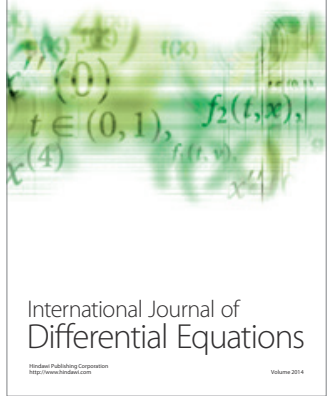
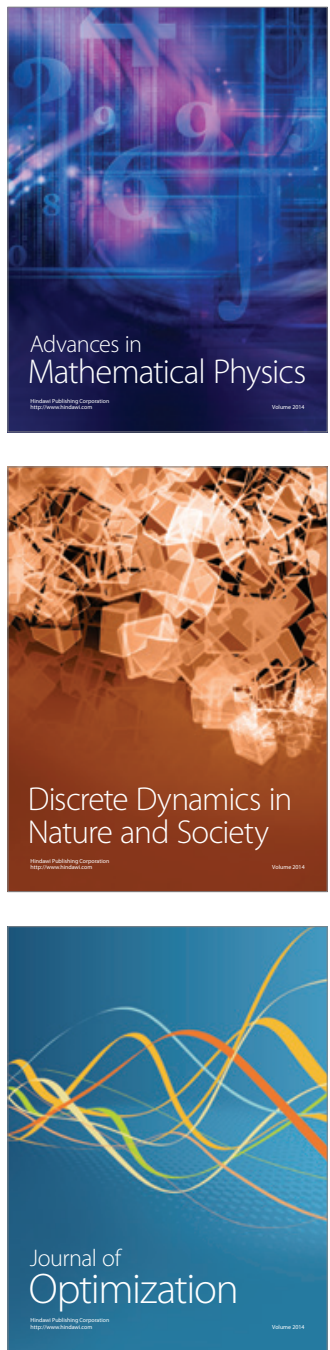